\title{
Awareness of Luxators in Dental Extraction Among Dentists
}

\author{
Chithambara Shathviha Palaniappan ${ }^{1}$ and Dhanraj Ganapathy ${ }^{2}$ \\ ${ }^{1}$ Saveetha Dental College, Saveetha Institute Of Medical and Technical Sciences, \\ Saveetha University, 162, Poonamallee High Road, Chennai, India \\ ${ }^{2}$ Professor and Head, Department of Prosthodontics, Saveetha Dental College and Hospitals \\ Saveetha Institute of Medical and Technical Sciences Saveetha University, Chennai, India
}

\begin{abstract}
Luxator looks like an elevator, but the tip of the instrument is significantly thinner and somewhat flatter than an elevator.One of the frequently occurring frustrating challenges when extracting teeth is breaking the tooth off at the bone level, or extracting a tooth that has decayed off at the bone level.The aim of this study is to determine the level of knowledge about the usage of luxator in dental extraction by the dentist and getting to the how aware they are about the use of luxator in their practice. The study was formulated as a questionnaire based observational study comprising 100 participants.All the subjects were requested to respond to a list of questions regarding luxators and their use in dental extraction by the dentists.More than $90 \%$ of the dentists are aware of the luxators and they prefer them during dental extraction.Luxator to be one of the most valuable instruments available for the situations of broken or decayed tooth .It is not a replacement for elevators. It is an augmentation for relatively easy removal of broken teeth. Soon after acquiring the instruments, dentists will find other uses for luxators
\end{abstract}

KEY WORDS: AWARENESS, LUXATOR, EXTRACTION, DENTISTS.

\section{INTRODUCTION}

The extraction of a tooth is probably the most traumatic event a patient can experience in the dental office, and if the extraction doesn't go smoothly, things can become quite stressful for the dentist as well.When the use of a simple surgical instrument can make the extraction process infinitely easier for both patient and dentist. (Weiss, Stern and Dym, 2011)Luxator helps the dentist to divide and conquer the forces retaining a tooth, making the extraction process an atraumatic extraction with Luxator Periotome is definitely a more predictable and stress-free process. (Tsirlis et al., 2015)The appropriate

\section{ARTICLE INFORMATION}

*Corresponding Author:dhanraj@saveetha.com

Received 11th June 2020 Accepted after revision 11th August 2020 Print ISSN: 0974-6455 Online ISSN: 2321-4007 CODEN: BBRCBA

Thomson Reuters ISI Web of Science Clarivate Analytics USA and Crossref Indexed Journal

\section{Clarivate ${ }_{\text {Analytics }}^{\text {Crossef }}$}

NAAS Journal Score 2020 (4.31) SJIF: 2020 (7.728)

A Society of Science and Nature Publication,

Bhopal India 2020. All rights reserved.

Online Contents Available at: http//www.bbrc.in/

Doi: http://dx.doi.org/10.21786/bbrc/13.7/24 size of Luxator is chosen to match the diameter of the root,and the angle of the blade is chosen to give the best access.(Kumar et al., 2013) The tip of the Luxator is gently inserted into the gingival margin, with the blade angled slightly toward the root surface.

This ensures that the Luxator enters the periodontal ligament between the crestal bone and the root. Once in the periodontal ligament, the Luxator is worked down the length of the root with a side-to-side rocking motion and steady axial pressure. (Arashiro et al., 2020) This motion first severs the periodontal fibers, and then as the blade is introduced further, the socket is dilated to allow an easier path of removal.(Bhusari et al., 2014) Finally, as the periodontal ligament is severed and the socket dilated, bleeding and air ingress overcome the vacuum that resists tooth removal.(Sharma et al., 2015) The Luxator should be inserted around as much of the circumference of the root as possible to evenly dilate the socket. Once this has been achieved, the final delivery of the tooth may be performed with forceps, although this 
is often not required with single-rooted teeth.(Tsirlis et al., 2015; Xu and Zhang, 2016)

When using a Luxator, the uniquely designed handle should sit neatly in the palm of your hand, cradled by your fingers and thumb, with the index finger extended toward the tip of the instrument . (Kang, Dym and Stern, 2009)This allows for precise control of the tip and prevents the risk of slipping. Excessive force should be avoided; the Luxator is a surgical instrument and should be used as such, not as an elevator.(Levitt, 2001) Most dentists have been taught to make a soft-tissue flap and remove bone on the facial side of such broken off teeth, allowing the teeth to be removed from the facial aspect. This bone removal is destructive, limits the possibility for placement of implants at a later date, and makes a permanent anatomical defect in the alveolar ridge.The use of luxators makes it esthetically acceptable.(Thomson, 1992; Kang, Dym and Stern, 2009) This study was undertaken in order to determine the level of knowledge about the usage of luxator in dental extraction by the dentist and getting to the how aware they are about the use of luxator in their practice.

Figure 1: The pie chart depicting the distribution of qualification of dentists participated in the survey.Blue colour indicates undergraduates (45\%). Green colour indicates postgraduates (26\%). Beige colour indicates oral and maxillofacial surgeons (29\%).

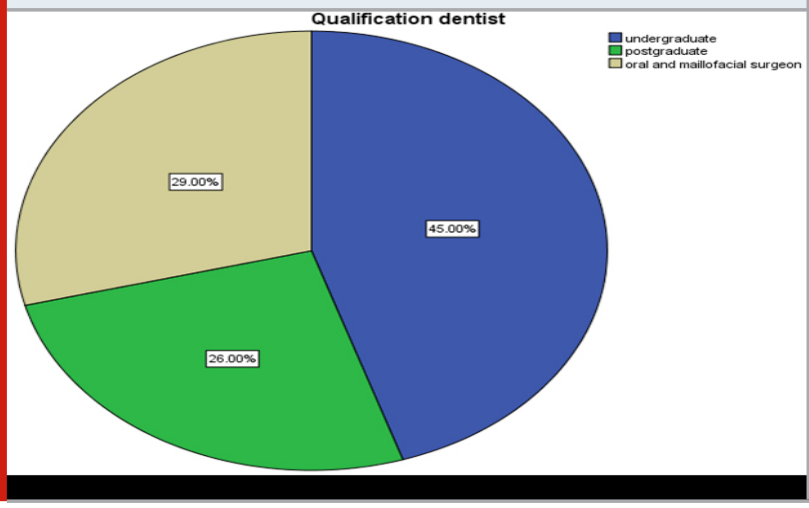

Figure 2: The pie chart depicts the use of luxator in clinical practice among the participants. Blue colour indicates yes ( 43\%). Green colour indicates no (21\%). Beige indicates sometimes (36\%).

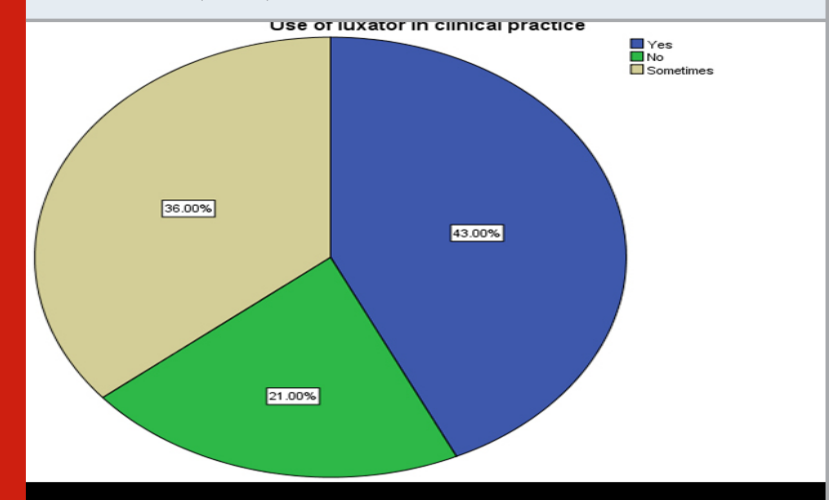

Previously our department has published extensive research on various aspects of prosthetic dentistry ('Evaluation of Corrosive Behavior of Four Nickelchromium Alloys in Artificial Saliva by Cyclic Polarization Test:An in vitro Study', 2017; Ganapathy, Kannan and Venugopalan, 2017; Jain, 2017a, 2017b; Ranganathan, Ganapathy and Jain, 2017; Ariga et al., 2018; Gupta, Ariga and Deogade, 2018; Anbu et al., 2019; Ashok and Ganapathy, 2019; Duraisamy et al., 2019; Varghese, Ramesh and Veeraiyan, 2019), this vast research experience has inspired us to research about awareness of luxator in dental extraction among dentists.

\section{MATERIAL AND METHODS}

The present study is an online based survey conducted among the dental students. The participants were the undergraduate students of BDS, postgraduates of oral and maxillofacial surgery and oral and maxillofacial surgeons.Questionnaires were prepared and distributed among undergraduates,postgraduates and oral maxillofacial surgeons through an online link from the google forms. The total number of participants was 100 dentists. Participation in this study was voluntary. The questionnaire contained 15 questions. Independent variables were demographics such as year of study of participants. Dependent variables were knowledge ,awareness about luxator in dental extraction and the dentists. Only the completed surveys were included for analysis. The collected results were entered in Microsoft excel. Data analysis was done using SPSS software 20.0. Statistics used for analysis was Descriptive statistics and comparison of variables were done using chi square test where $\mathrm{p}<0.05$, statistically significant .

Figure 3: The pie chart depicts the usage of luxator in the extraction treatment. Blue colour indicates that the luxator is used when the tooth is broken at bone level $(82 \%)$. Green colour indicates that the luxator is used in normal extraction treatment (18\%)

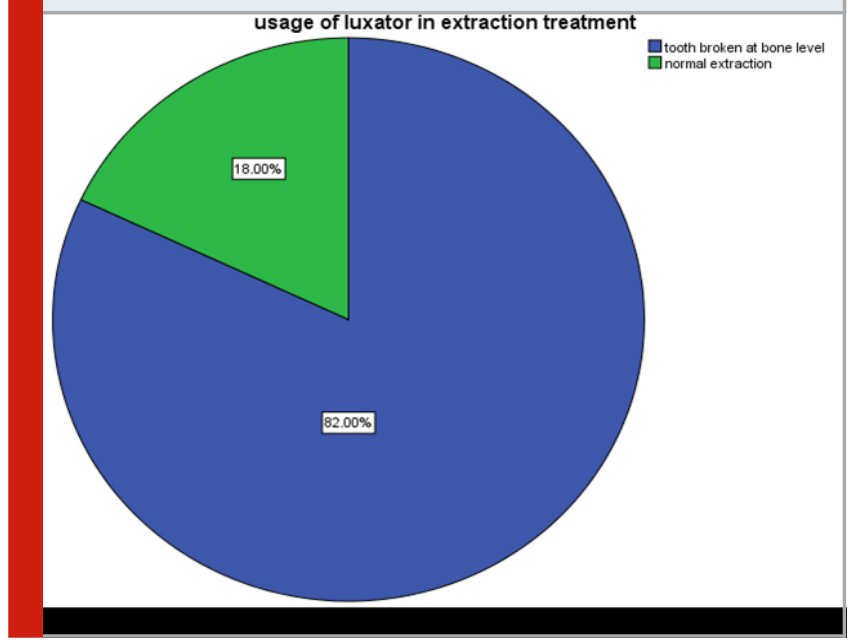

RESULTS AND DISCUSSION

The participants of the survey were the undergraduate 
students of BDS , postgraduates of oral and maxillofacial surgery and oral and maxillofacial surgeons. The survey results obtained by the statistical analysis is discussed here, the participation by undergraduates is $45 \%$ and postgraduates is $26 \%$ and oral and maxillofacial surgeons is $29 \%$. (Figure 1). The use of luxator in clinical practice by the participants shows that $43 \%$ use luxator and 21\% of the participants do not use the luxator and 36\% of participants use luxator sometimes during the dental extraction. (Figure 2). The luxator is used when the tooth is broken at the bone level $82 \%$ and the luxator is used in normal extraction treatment by the respondents is $18 \%$. (Figure 3). The position of luxator placed between tooth surface and mesial and distal supporting bone is $84 \%$, the luxator placed between tooth surface and palatal bone is $6 \%$ and the luxator placed between tooth surface and thin piece of bone is $10 \%$ by the respondents (Figure 4).

Figure 4: The pie chart depicts the position of luxator in clinical practice during extraction. Blue colour indicates that the luxator is placed between tooth surface mesial and distal supporting bone (84\%). Green colour indicates that the luxator is placed between tooth surface and palatal bone $(6 \%)$. Beige colour indicates that the luxator is placed between the tooth surface and thin piece of bone (10\%)

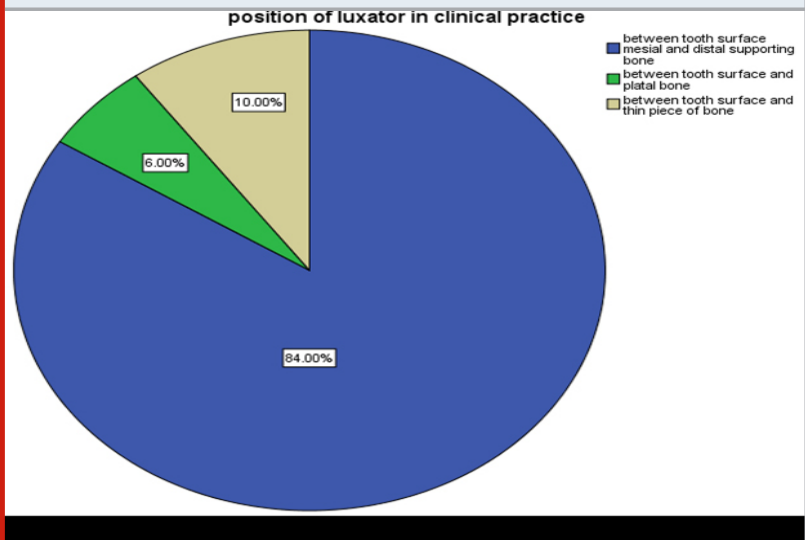

Figure 5: The pie chart depicts the preference of luxator over an elevator in dental extraction. Blue colour indicates yes $(57 \%)$.Green colour indicates no (27\%).Beige colour indicates sometimes $(16 \%)$.

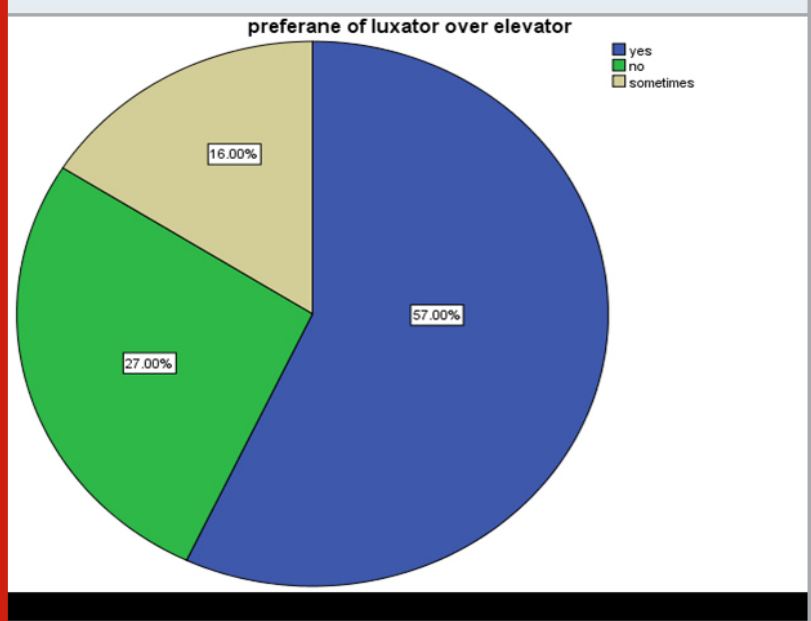

Preference of luxator over an elevator 57\% agree the use of luxator ,27\% do not agree and 16\% of the participants sometimes prefer luxator over elevator. The reason to choose a luxator over an elevator is that the tip is thin $69 \%$, the tip is flat $20 \%$ and the luxator used in normal extraction by the participants is $11 \%$ (Figure 6). 82\% of the participants agree that luxator is used by them when the tooth is broken at bone level and 18\% disagree. ( Figure 7). $76 \%$ of the participants agree that use of luxator is time consuming $14 \%$ of the participants assume that it is less time consuming. (Figure 8). $87 \%$ of the participants agree that use of luxator increases the potential implant placement and 13\% disagreed.The present study consensus with (Sharma et al., 2015) (Figure 9).The awareness on use of luxator in dental extraction among the participants is higher with $92 \%$ and $8 \%$ are unaware. ( Figure 10). The preference of luxator in dental instruments used in dental extraction by the dentists is $34 \%$. (Figure 11).

FIgure 6: The pie chart depicts the reason to use luxator over an elevator in dental extraction. Blue colour indicates that the tip is thin (69\%). Green colour indicates that the tip is flat $(20 \%)$. Beige colour indicates that it is used for normal extraction $(11 \%)$

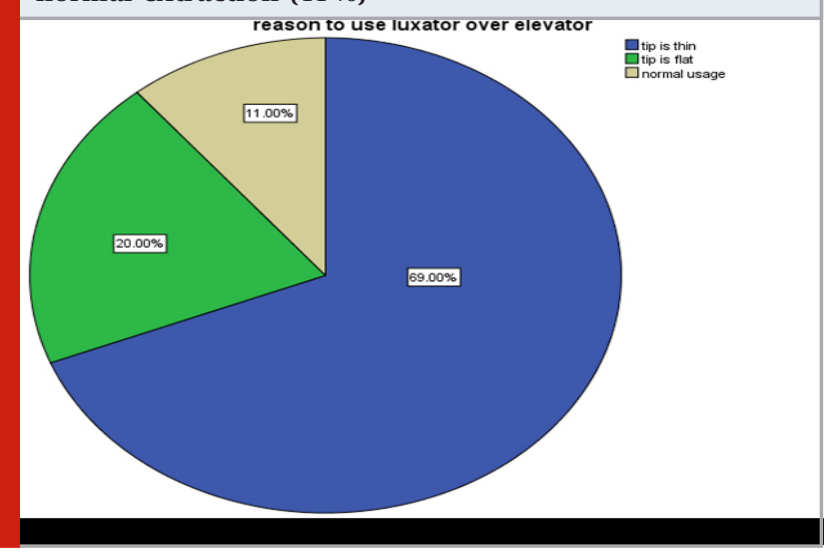

Figure 7: The pie chart depicts the use of a luxator when the tooth is broken at the bone level. Blue colour indicates yes ( $82 \%)$. Green colour indicates no (18\%)

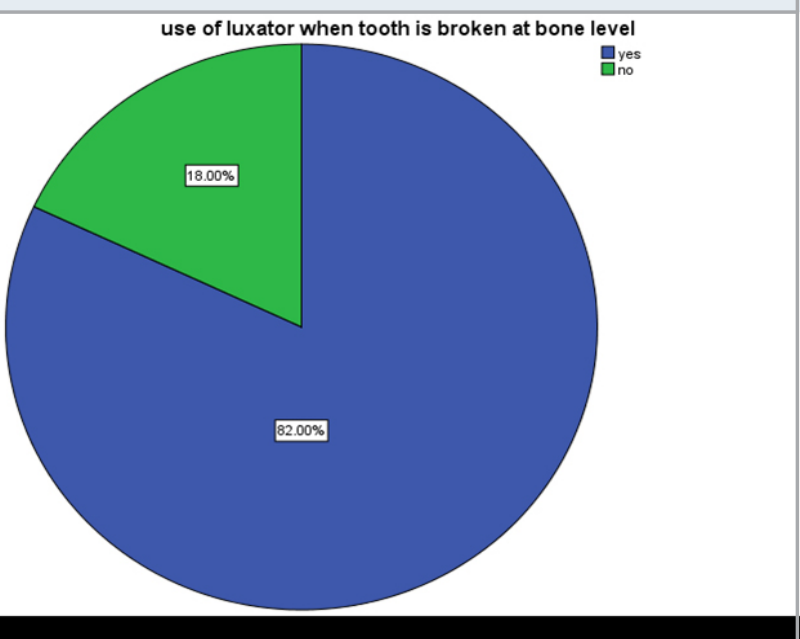


Figure 8: The pie chart depicts the time span of using a luxator in dental extraction. Blue colour indicates that it is time consuming (76\%). Green colour indicates that it is less time consuming (14\%). Beige colour indicates that it depends on the tooth and use by the dentist (10\%)

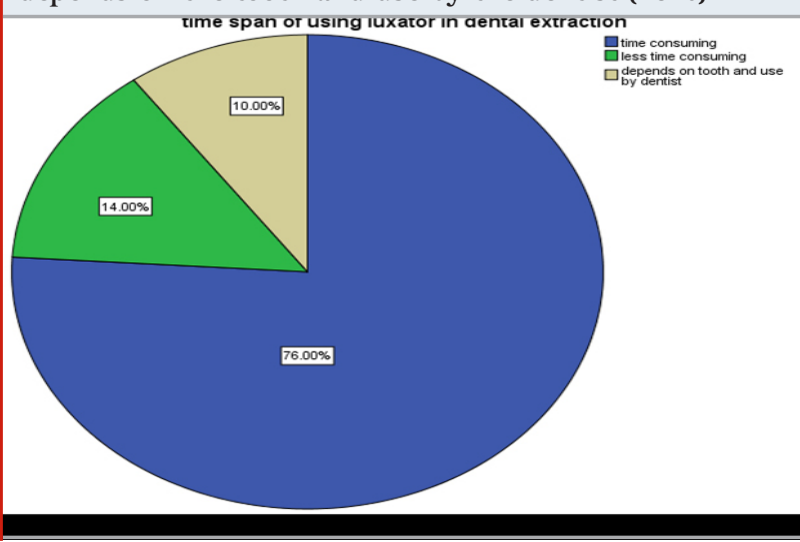

Figure 9: The pie chart depicts that luxator helps in potential implant placement. Blue colour indicates yes (87\%). Green colour indicates no (13\%)

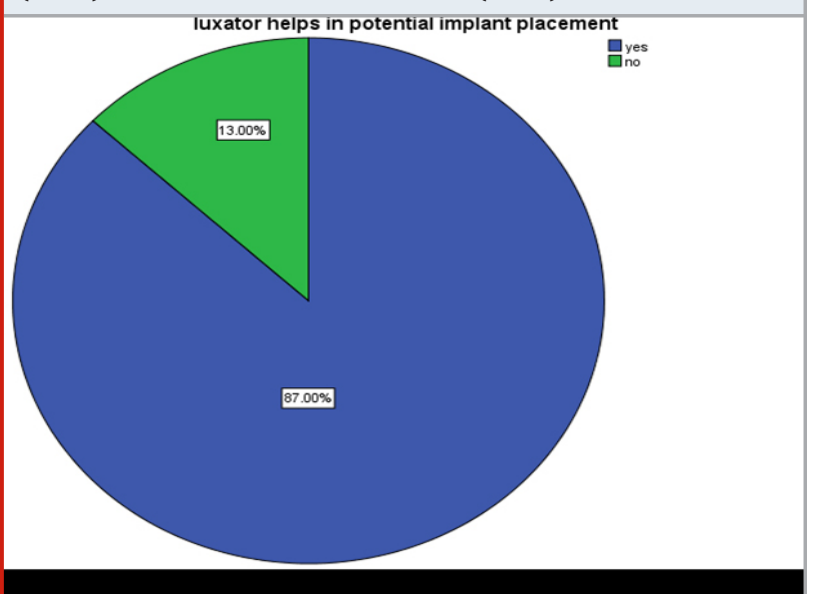

Figure 10: The pie chart depicts the awareness and knowledge on luxator among the participants. Blue colour indicates that they are aware (92\%). Green colour indicates that they are not aware (8\%)

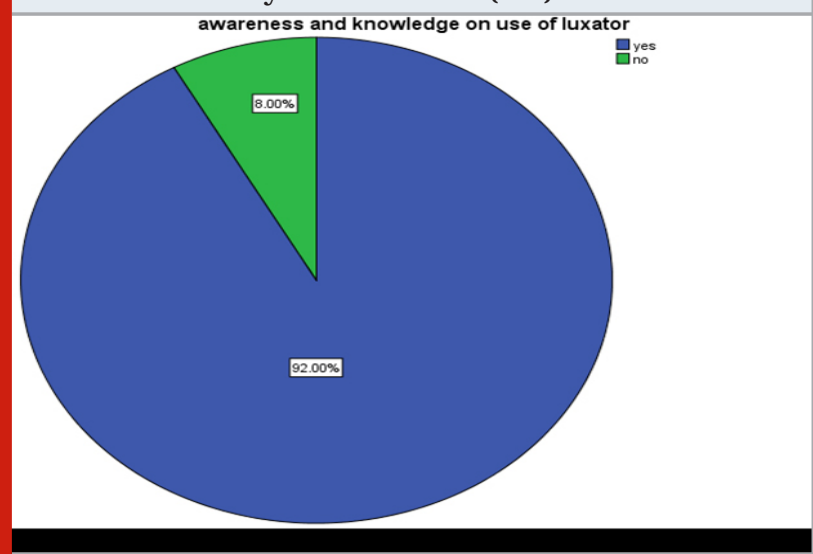

Figure 11: The pie chart depicts the preference of dental instruments used in extraction by the dentists. Blue colour indicates luxator (34\%). Green colour indicates elevator ( 56\%). Beige colour indicates forceps (10\%)

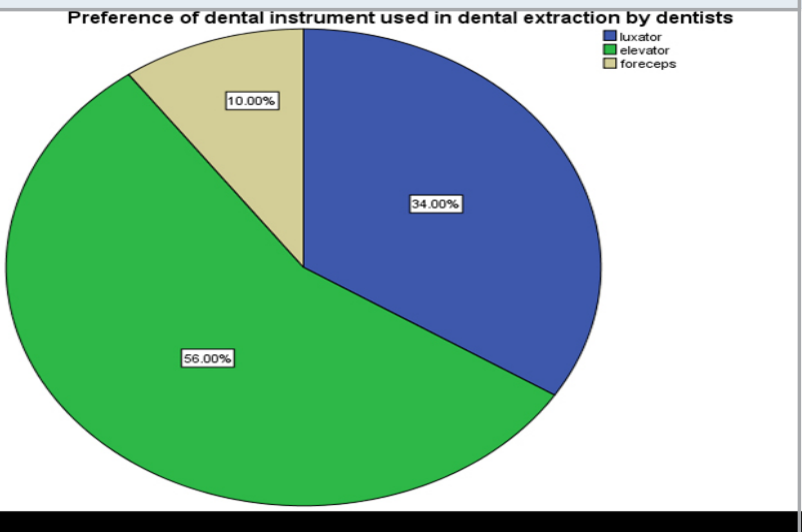

Comparison of qualification of dentist and the frequency showing the use of luxator increases the chance of implant placements was done and majority of participants agreed that the use of luxator increases the chance of implant placement (62\%). This was found to be statistically significant where $p=0.02$.(Figure 12 ). Comparison of qualification of dentist and the frequency showing the use of luxator in clinical practice. The use of luxator among the undergraduates is more (25\%) compared to others. This was found to be statistically not significant where $\mathrm{p}=0.38$. (Figure 13).

Figure 12: The bar graph depicts the comparison of qualification of dentist and the frequency showing the use of luxator increases the chance of implant placement. $\mathrm{X}$ axis denoted the qualification of dentist and $\mathrm{Y}$ axis denotes number of participants. Blue colour depicts the participants who agree that use of luxator increases the chance of implant placement and green colour depicts the participants who do not agree and beige colour depicts that they might agree nor disagree. Majority of the participants agreed that use of luxator increases the chance of implant placement ( 62\%). This was found to be statistically significant. Chi square test, $\mathrm{p}=0.02$

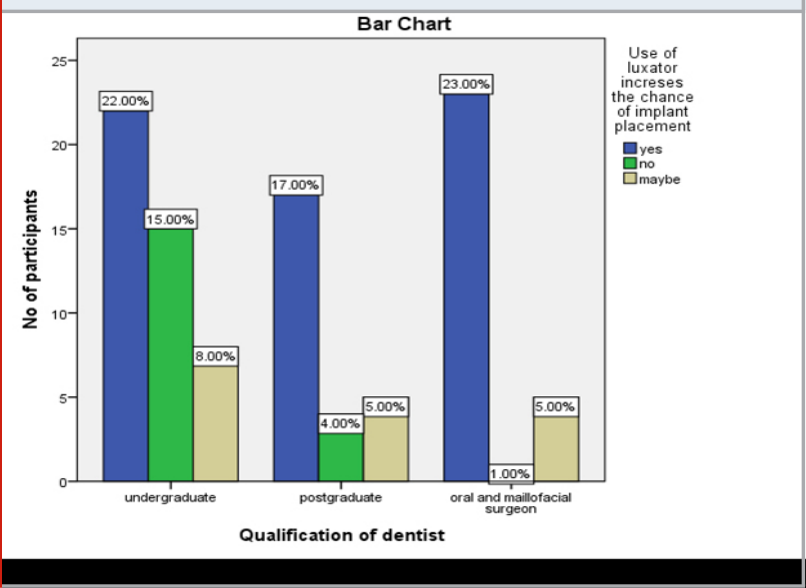


Figure 13: The bar graph depicts the comparison of qualification of dentist and the frequency of use of luxator in clinical practice. $\mathrm{X}$ axis denotes the qualification of dentist and the $\mathrm{Y}$ axis denotes the number of participants. Blue colour indicates that the dentists use luxator in their clinical practice and green colour indicates that dentists do not use luxator and beige colour indicates that the dentists use luxator sometimes during their clinical practice. The use of luxator among the undergraduates is more (25\%) compared to others. This was found to be statistically not significant. (Chi square test,p=0.38)

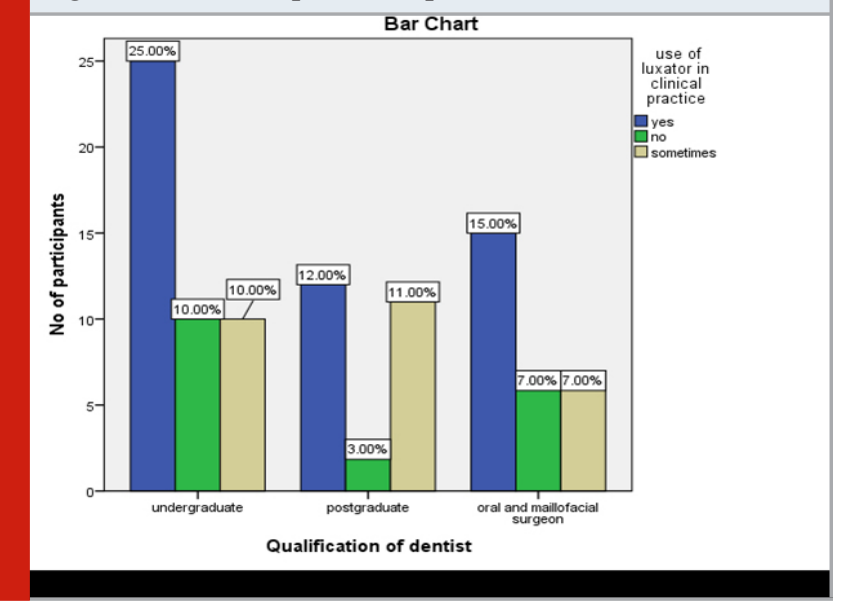

CONCLUSION

This survey aims in creating awareness among dentists about the use of luxator in dental extraction during clinical practise. It also creates awareness about the complications and advantages of using luxator in dental extraction.From the results of the survey it is clear that most of the dentists are well aware of the use of luxator in clinical practice and also proper knowledge about the complications and advantages of using luxator in dental extraction.

\section{ACKNOWLEDGEMENTS}

The authors would like to thank the study participants for their participation and kind cooperation throughout the study.

Conflict of Interest: The authors declare that there were no conflicts of interest in the present study

\section{REFERENCES}

Anbu, R. T. et al. (2019) 'Comparison of the Efficacy of Three Different Bone Regeneration Materials: An Animal Study', European journal of dentistry, 13(1), pp. 22-28. doi: 10.1055/s-0039-1688735.

Arashiro, F. N. et al. (2020) 'Dentinal microcracks on freshly extracted teeth: the impact of the extraction technique', International endodontic journal, 53(4), pp. 440-446. doi: 10.1111/iej.13239.

Ariga, P. et al. (2018) 'Determination of Correlation of
Width of Maxillary Anterior Teeth using Extraoral and Intraoral Factors in Indian Population: A Systematic Review', World Journal of Dentistry, 9(1), pp. 68-75. doi: 10.5005/jp-journals-10015-1509.

Ashok, V. and Ganapathy, D. (2019) 'A geometrical method to classify face forms', Journal of oral biology and craniofacial research, 9(3), pp. 232-235. doi: 10.1016/j.jobcr.2019.06.001.

Bhusari, P. et al. (2014) 'ATRAUMATIC SURGICAL EXTRUSION OF COMPLETE CROWN FRACTURED TOOTH USING PERIOTOME IN ESTHETIC ZONE: REPORT OF THREE CASES', Journal of Evolution of Medical and Dental Sciences, pp. 6550-6558. doi: 10.14260/jemds/2014/2780.

Duraisamy, R. et al. (2019) 'Compatibility of Nonoriginal Abutments With Implants: Evaluation of Microgap at the Implant-Abutment Interface, With Original and Nonoriginal Abutments', Implant dentistry, 28(3), pp. 289-295. doi: 10.1097/ID.0000000000000885.

Evaluation of Corrosive Behavior of Four Nickelchromium Alloys in Artificial Saliva by Cyclic Polarization Test:An in vitro Study' (2017) World Journal of Dentistry, 8(6), pp. 477-482. doi: 10.5005/ jp-journals-10015-1490.

Ganapathy, D. M., Kannan, A. and Venugopalan, S. (2017) 'Effect of Coated Surfaces influencing Screw Loosening in Implants: A Systematic Review and Metaanalysis', World Journal of Dentistry, pp. 496-502. doi: 10.5005/jp-journals-10015-1493.

Gupta, P., Ariga, P. and Deogade, S. C. (2018) 'Effect of Monopoly-coating Agent on the Surface Roughness of a Tissue Conditioner Subjected to Cleansing and Disinfection: A Contact Profilometric Study', Contemporary clinical dentistry, 9(Suppl 1), pp. S122S126. doi: 10.4103/ccd.ccd_112_18.

Jain, A. R. (2017a) 'Clinical and Functional Outcomes of Implant Prostheses in Fibula Free Flaps', World Journal of Dentistry, 8(3), pp. 171-176. doi: 10.5005/jp-journals10015-1433.

Jain, A. R. (2017b) 'Prevalence of Partial Edentulousness and Treatment needs in Rural Population of South India', World Journal of Dentistry, 8(3), pp. 213-217. doi: 10.5005/jp-journals-10015-1440.

Kang, J., Dym, H. and Stern, A. (2009) 'Use of the Powertome Periotome to Preserve Alveolar Bone During Tooth Extraction - A Preliminary Study', Oral Surgery, Oral Medicine, Oral Pathology, Oral Radiology, and Endodontology, pp. 524-525. doi: 10.1016/j. tripleo.2009.06.070.

Kumar, P. et al. (2013) 'Atraumatic surgical extrusion using periotome in esthetic zone: A case series', Journal of Conservative Dentistry, p. 175. doi: 10.4103/09720707.108213. 
Levitt, D. (2001) 'Atraumatic extraction and root retrieval using the periotome: a precursor to immediate placement of dental implants', Dentistry today, 20(11), pp. 53-57. Available at: https://www.ncbi.nlm.nih.gov/ pubmed/11715649.

Ranganathan, H., Ganapathy, D. M. and Jain, A. R. (2017) 'Cervical and Incisal Marginal Discrepancy in Ceramic Laminate Veneering Materials: A SEM Analysis', Contemporary clinical dentistry, 8(2), pp. 272-278. doi: 10.4103/ccd.ccd_156_17.

Sharma, S. D. et al. (2015) 'Periotome as an Aid to Atraumatic Extraction: A Comparative Double Blind Randomized Controlled Trial', Journal of maxillofacial and oral surgery, 14(3), pp. 611-615. doi: 10.1007/ s12663-014-0723-8.

Thomson, P. J. (1992) 'Minimising trauma in dental extractions: the use of the periotome', British dental journal, 172(5), p. 179. doi: 10.1038/sj.bdj.4807815.

Tsirlis, A. T. et al. (2015) 'A technique for atraumatic root extraction, immediate implant placement and loading in maxillary aesthetic zone', Oral Surgery, pp. 102-110. doi: 10.1111/ors.12131.

Varghese, S. S., Ramesh, A. and Veeraiyan, D. N. (2019) 'Blended Module-Based Teaching in Biostatistics and Research Methodology: A Retrospective Study with Postgraduate Dental Students', Journal of dental education, 83(4), pp. 445-450. doi: 10.21815/ JDE.019.054.

Weiss, A., Stern, A. and Dym, H. (2011) 'Technological advances in extraction techniques and outpatient oral surgery', Dental clinics of North America, 55(3), pp. 501-13, viii. doi: 10.1016/j.cden.2011.02.008.

$\mathrm{Xu} \mathrm{F}$. and Zhang H.-X. (2016) '[Comparison of minimally invasive extraction and traditional method in the extraction of impacted mandibular third molar]', Shanghai kou qiang yi xue = Shanghai journal of stomatology, 25(5), pp. 613-616. Available at: https:// www.ncbi.nlm.nih.gov/pubmed/28116438. 